\title{
Evolving Open-Source Technologies Offer Options for Remote Sensing and Monitoring in Agriculture
}

\author{
Daniel K. Fisher ${ }^{1 *}$, Reginald S. Fletcher ${ }^{2}$, Saseendran S. Anapalli1 \\ ${ }^{1}$ United States Department of Agriculture, Agricultural Research Service, Sustainable Water Management Research Unit, \\ Stoneville, Mississippi, USA \\ ${ }^{2}$ United States Department of Agriculture, Agricultural Research Service, Crop Production Systems Research Unit, Stoneville, \\ Mississippi, USA \\ Email: ^daniel.fisher@usda.gov, reginald.fletcher@usda.gov, saseendran.anapalli@usda.gov
}

How to cite this paper: Fisher, D.K., Fletcher, R.S. and Anapalli, S.S. (2020) Evolving Open-Source Technologies Offer Options for Remote Sensing and Monitoring in Agriculture. Advances in Internet of Things, 10, 1-10.

https://doi.org/10.4236/ait.2020.101001

Received: September 26, 2019

Accepted: November 4, 2019

Published: November 7, 2019

Copyright $\odot 2020$ by author(s) and Scientific Research Publishing Inc. This work is licensed under the Creative Commons Attribution International License (CC BY 4.0).

http://creativecommons.org/licenses/by/4.0/

\begin{abstract}
A variety of sensing and monitoring systems have been developed based on the concept of open-source and on open-source hardware and software components. Availability and relatively low cost of hardware components and availability and ease of use of software components allow access to sensing and monitoring technologies that were previously unattainable to many potential users. Advances in electronic monitoring and evolving cellular communications technologies are increasingly offering more, simpler, and less expensive options for remote monitoring. Due to the near-future cessation of $2 \mathrm{G}$ and $3 \mathrm{G}$ cellular network services, however, many existing monitoring systems will need to be redesigned to operate on alternative cellular networks. A soil-moisture monitoring system was developed incorporating updated open-source Arduino microcontrollers and the recently introduced LTE Cat-M1 cellular network to transmit sensor measurements via the cellular network for access on an internet website. The monitoring system costs approximately US $\$ 130$ to construct the electronic circuitry and less than US\$1 per month for cellular network access and data transmission. Data were transmitted with a $95 \%$ success rate, and the monitoring system operated continuously throughout an entire crop growing season with no battery recharge or maintenance requirements. The design and operation of the monitoring system can serve as a basis for other remote monitoring systems.
\end{abstract}

\section{Keywords}

Arduino, Microcontrollers, Cellular, Soil Moisture, Sensors, Internet of Things 


\section{Introduction}

A variety of sensing and monitoring systems have been developed based on the concept of open-source and on open-source hardware and software components. The concept of open sharing of ideas and designs, collaborative efforts, and supportive community has enabled researchers and others to develop unique and custom devices to satisfy specific requirements and needs [1] [2]. Availability and relatively low cost of hardware components and availability and ease of use of software components allow access to sensing and monitoring technologies that were previously unattainable to many potential users.

An open-source hardware and software project that has been popular with developers is the Arduino microcontroller development platform (https://www.arduino.cc). The Arduino hardware consists of a microcontrollerbased development board designed to allow convenient access to the microcontroller's various features, including input/output pins, analog-to-digital converters, analog and digital communications protocols, timers, and non-volatile memory. The many features allow a user to interface and interact with external components, such as sensors, motors, displays, data-storage devices, and wireless data-transmission devices, to create custom monitoring and control systems. The Arduino Integrated Development Environment (IDE) software provides a programming environment for the user to write and upload programs to the microcontroller to control the operation of the system.

A key component of a sensing and monitoring system is the accessibility of the information collected by the system. While the information can be collected and stored at the location of the system, accessing the location and retrieving the data can be time-consuming and inconvenient, especially for remotely located systems. Many wireless technologies exist and have been explored for transmitting data [3] [4] [5], but rapid expansion and widespread coverage of modern cellular networks have resulted in a communications network that is accessible to many in most parts of the world. Agricultural researchers have begun deploying open-source monitoring systems and cellular communications to aid in efforts related to irrigation and water management [6] [7] [8], soil moisture monitoring [9] [10] [11] [12], water use [13], and weather and rainfall monitoring [14] [15].

As the user base of the Arduino microcontroller platform has expanded, in terms of the interests and number of users and the sophistication of Arduino-based projects, the capabilities of the microcontroller are becoming a limiting factor. The original Arduino development board included a specific microcontroller and a standardized physical size and microcontroller pin configuration. The Atmel 8-bit microcontroller had $32 \mathrm{~KB}$ of Flash memory for program storage and $2 \mathrm{~KB}$ of SRAM for temporary storage, a $5 \mathrm{~V}$ operating voltage, and a clock speed of $16 \mathrm{MHz}$. While approximately 20 microcontroller input/output pins were available to the user, each individual pin was limited in the features available. Variations of the development board were introduced over time, af- 
fecting physical size and pin configurations, and employing different microcontrollers, with most remaining similar in specifications and features to those of the original 8-bit design.

The Arduino platform received a significant increase in capabilities with the introduction of the Arduino Zero development board. The original microcontroller was replaced with an Atmel ATSAMD21G18 32-bit ARM Cortex-M0+ processor, with $256 \mathrm{~KB}$ of Flash memory, $32 \mathrm{~KB}$ of SRAM, 3.3V operation, and a processing speed of $48 \mathrm{MHz}$. The greatly expanded memory allowed for larger, more advanced programs and helped avoid problems of temporary memory stack overflows during program operation. Additional feature upgrades included the ability to use almost all input/output pins for multiple capabilities, including pulse-width modulation, external interrupt inputs, two serial communications ports, and higher-resolution 12-bit analog-to-digital channels. The Arduino IDE also received periodic updates to support this upgraded and many other microcontrollers.

The cellular communications networks have also continued to evolve, offering expanded capabilities. Rapid expansion of Machine-to-Machine (M2M) communications, increasingly referred to as the Internet of Things (IoT), enables objects in the environment to gather information, recognize events, and detect changes in their surroundings and share these via the communications network [16]. As older cellular technologies, such as $2 \mathrm{G}$ and $3 \mathrm{G}$ networks, are being retired and will no longer be available, they are being replaced by new technologies such as LTE Cat-M1. LTE Cat-M1 is an extension of and compatible with the existing LTE cellular network, requiring minimal investment by cellular network providers to upgrade their networks and offer dedicated M2M and IoT services (https://www.3gpp.org). The LTE Cat-M1 network is designed to support lowdata-rate, low-power IoT applications and offer widespread support and communications for distributed monitoring and control systems.

While projects such as those cited above have brought remote monitoring to agricultural endeavors, the technologies and components involved are evolving, requiring updated designs and instrumentation. Due to the near-future cessation of $2 \mathrm{G}$ and $3 \mathrm{G}$ cellular network services, many existing monitoring systems will need to be redesigned to operate on newer or alternative cellular networks. The objective of the work described here was to develop a remote sensing and monitoring system incorporating updated open-source components and evolving cellular communications for use in agricultural applications.

\section{Materials and Methods}

A monitoring system was developed to measure and transmit soil-moisture sensor measurements for use in determining the proper timing of irrigation applications and for monitoring water resources in the root zone. The system was comprised of open-source hardware and software components and was designed to allow internet access of sensor measurements from remotely located agricul- 
tural fields.

The monitoring system hardware consisted of a microcontroller-based circuit, four soil-moisture sensors, and a cellular modem. A SAMD21 Mini Development Board (Sparkfun Electronics, Niwot CO USA; https://sparkfun.com) was selected for its small physical size, hardware serial communications, and lowpower sleep capabilities. An electrical circuit, shown in Figure 1, was designed to interface up to four Watermark 200-SS soil-moisture sensors (Irrometer Company, Inc., Riverside, CA USA; https://www.irrometer.com) to the microcontroller via four resistor voltage-divider circuits. Each voltage divider circuit was formed with a fixed-value resistor and a Watermark sensor, which behaves electrically as a variable resistor. When the voltage-divider was activated by applying voltage to the circuit, the voltage level at the center of the two resistors was measured with one of the microcontroller's built-in analog-to-digital converter channels.

The Botletics LTE Cat-M1 Cellular Shield (Botletics, Atlanta, GA USA; https://www.botletics.com) incorporates a SIM7000 LTE Cat-M1 cellular modem (SIMCom, Shanghai, China; http://www.simcom.com) and auxiliary electronic components into a standard-sized Arduino-compatible circuit board. The Botletics shield was interfaced with the microcontroller to enable access to the cellular shield's power/control functions and serial communications. This enabled the microcontroller to turn the modem on, transfer sensor data to the cellular modem, configure the modem for cellular network access, transmit sensor data to an internet website, and turn the modem off.

Access to the LTE Cat-M1 cellular network was provided via a Hologram.io SIM card and data plan (Hologram, Chicago, IL USA; https://hologram.io). The

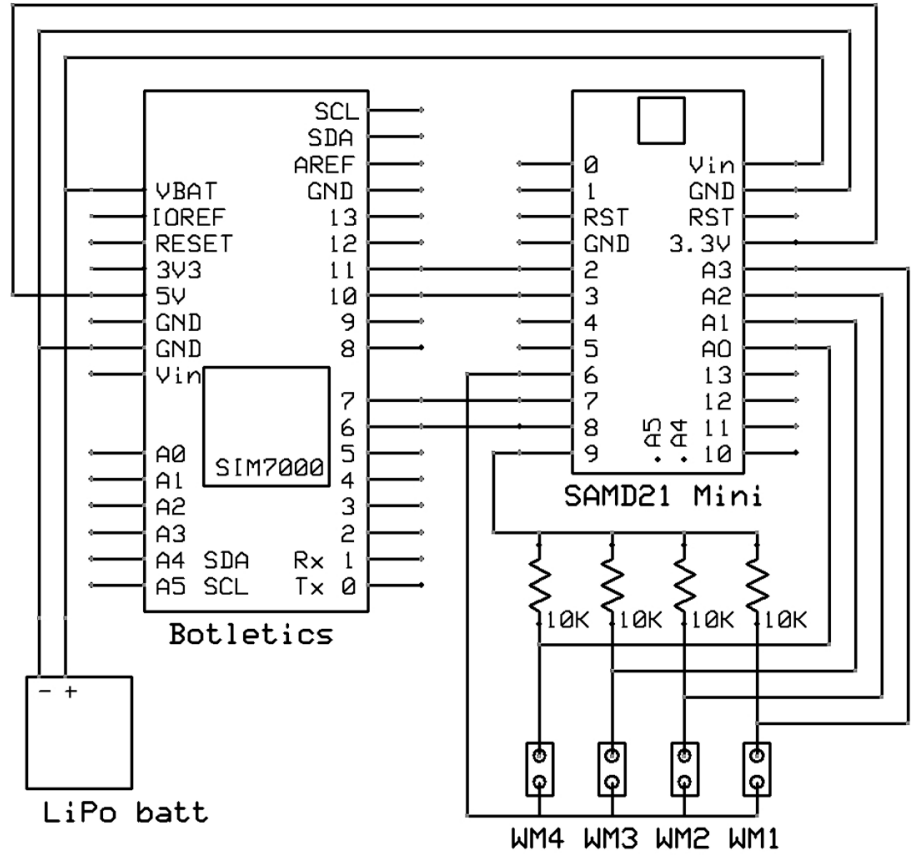

Figure 1. Electrical schematic. 
Hologram SIM card allows LTE Cat-M1 network access in almost every country and provides inexpensive (US $\$ 0.60 /$ month for $1 \mathrm{MB}$ data transfer) and convenient payment and SIM card/data use account management. Higher data-transfer amounts are available if needed at an additional cost of US $\$ 0.40$ per $1 \mathrm{MB}$.

A hardware circuit board was then fabricated based on the electrical schematic. The circuit was built on a Sparkfun Prototyping Shield (Sparkfun Electronics) with size and pin configuration compatible with those of the Botletics cellular shield. Female headers, spring terminal blocks, fixed-value resistors, and jumper wires were soldered to the prototyping shield to form the electrical circuit. Male header pins were soldered to the SAMD21 Mini development board to mate with the female headers on the prototyping shield, and male and female headers were soldered to the prototyping and cellular shields to allow them to mate with each other. A rechargeable lithium-polymer battery connected to the cellular shield to power the entire circuit. The hardware components, along with sources and estimated costs, are listed in Table 1, and the completed hardware circuit components of the monitoring system are shown in Figure 2.

Table 1. List of material and approximate costs.

\begin{tabular}{cccc}
\hline Main components & Model number & Supplier & Cost (US\$) \\
\hline Microcontroller & SAMD21 Mini & Sparkfun Electronics & 21 \\
Cellular shield & LTE CAT-M1 & Botletics & 70 \\
Rechargeable battery & 3.7 V LiPoly, 2500 mAh & Adafruit Industries & 15 \\
Weatherproof enclosure & NBF-32004 & Digikey Electronics & 10 \\
Miscellaneous (prototype shield, terminal blocks, resistors, SIM card) & 14 \\
Total & & & 130 \\
Cellular data plan & $1 \mathrm{MB} / \mathrm{month}$ & Hologram.io & $1 /$ month \\
\hline
\end{tabular}

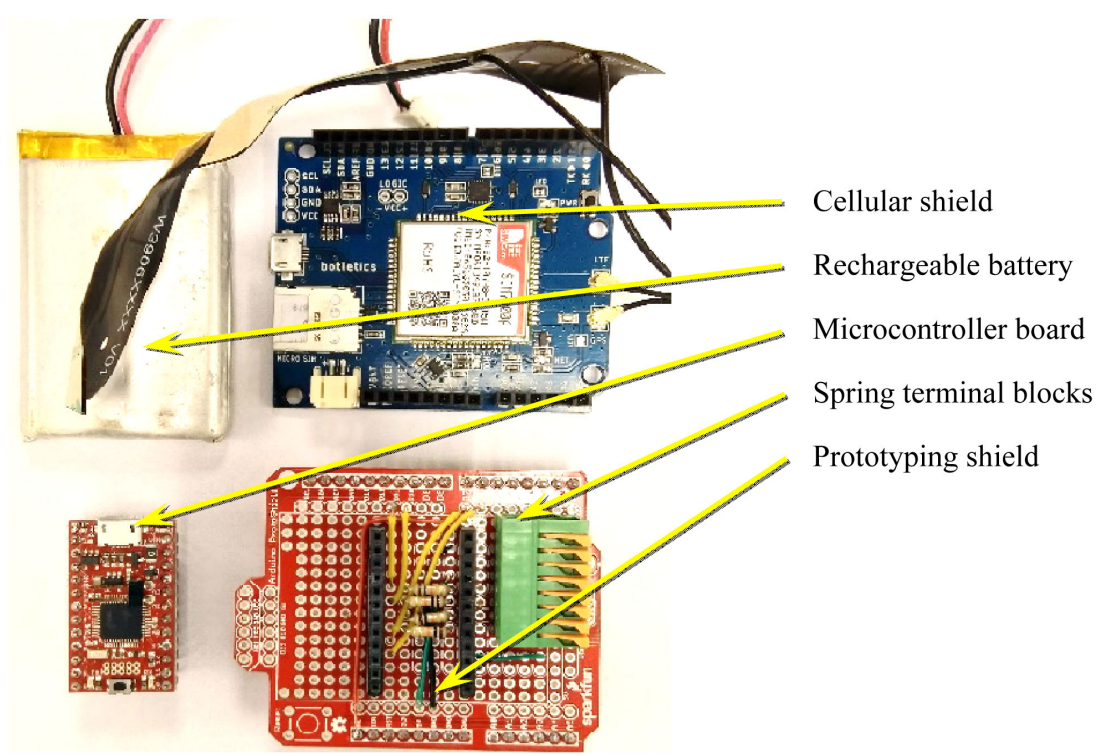

Figure 2. Soil-moisture sensor monitoring system hardware components. 
A microcontroller program was written to manage all sensor-measurement, data-transfer, and power-management functions of the hardware circuit. The program was written using the open-source Arduino IDE, which was downloaded from the Arduino project website (https://www.arduino.cc/) and installed on a personal computer. The program began with instructions for including program modules (libraries) containing routines for interfacing with hardware devices, including the cellular modem and the microcontroller's internal real-time clock. The devices were initialized, and program variables and input/output pins configured.

The main program section was then entered, which consisted of calls to program subroutines. Separate subroutines were written for accomplishing various tasks; making moisture-sensor measurements, transmitting data via cellular modem, and putting the circuit into a low-power sleep mode. Operation of the program and its subroutines is described briefly below. The microcontroller program is open-source and freely available by contacting the authors.

Soil-moisture measurements are obtained by reading each sensor individually. The voltage-divider circuit is energized by setting one end pin of the circuit high and the other end pin low, causing electricity to flow through the circuit with one polarity, and the center voltage is read with an analog-to-digital converter. The polarity is then reversed by reversing the voltage settings on the two end pins, and the center voltage read again. This is repeated five times to avoid long-term polarization of, and damage to, the sensors, and to obtain an average measurement. The resistance of the sensor, which varies in proportion to the moisture level of the sensor, is calculated using the standard voltage-divider equation. A calibration equation is then applied to the calculated resistance to estimate the water potential of the soil (see [2] for more detailed information about the circuit and calibration).

The microcontroller then turns on and establishes communication with the cellular modem to transmit data to an internet-based data-hosting website, described below. The modem registers with the cellular network and enables cellular data services and reads the voltage of the circuit's battery. The microcontroller assembles the sensor data and battery voltage into the proper format for internet traffic and performs the data transmission. The status of the transmission is monitored, and if unsuccessful, the modem makes up to three attempts to send the data successfully.

Following successful data transmission, the program terminates internet and cellular data services. The microcontroller's internal real-time clock is reset, and the next measurement time is specified, and the microcontroller, sensor circuit, and cellular modem are put into a low-power sleep mode. The circuit remains in the low-power mode until the next measurement time, a two-hour interval, when an internal alarm is sent, and the microcontroller is awakened. It is powered on and the measurement and data-transmission process is repeated.

To provide timely and convenient access to sensor data, the data are sent to an internet-based data-hosting service. The service, ThingSpeak 
(https://thingspeak.com), allows a user to establish and configure a webpage for viewing of a data stream from any location using a standard web browser. To encourage initial exploration of the use of web-based data-hosting services, ThingSpeak allows a user to have access to the website at no cost. To set up a webpage, the user creates a log-in account and configures the incoming data stream (number of data values, description of the data) and output data graphs (length of data series, graphical features). A unique channel number is assigned to the webpage, used to view the specific webpage, and a keycode is generated that is used to upload data to the webpage. To upload data, a website URL is assembled in a specific format containing the address of the data-hosting website, the webpage's keycode, and data-stream values. The URL is sent and when the webpage receives the data stream, the output data graphs are updated immediately. The data streams are stored on the ThingSpeak website and the user can download all data values for use elsewhere or for further analysis at any time.

\section{Field Deployment}

Soil-moisture monitoring systems developed in this study were deployed in agricultural fields under cultivation by the United States Department of Agriculture's Agricultural Research Service, Sustainable Water Management Research Unit, Stoneville, MS, USA during the 2019 growing season to monitor moisture conditions in the root zone and to determine the timing of irrigation applications. At each deployment location, soil-moisture sensors were installed at four depths; 1, 2, 3, and $4 \mathrm{ft}$ (approximately 30,60, 90, and $120 \mathrm{~cm}$ ) below the soil surface. A wooden stake was driven into the soil near the sensors, and a weatherproof plastic enclosure was attached to the stake at a height of approximately $20 \mathrm{~cm}$ above the soil surface. The sensor wires were attached to the monitoring system circuit board via the spring terminal blocks, the battery was connected to the circuit, and the completed monitoring circuitry was placed inside the weatherproof enclosure.

A sample of soil-moisture data collected over a 30-day period at one location is shown on that location's ThingSpeak webpage in Figure 3. The monitoring systems transmitted sensor data at 2-hr intervals throughout the2019 growing season, with approximately $95 \%$ successful data transmissions. The low-power sleep mode of the circuit and the infrequent data transmissions enabled the monitoring system to operate continuously throughout the growing season, approximately 4 months, with no maintenance or battery replacement required. The low amounts of data transmitted each month totaled less than $1 \mathrm{MB}$, the amount included in the Hologram.io data plan's standard monthly allowance, resulting in a monthly charge of US $\$ 0.60$ for each monitoring system.

\section{Conclusions}

A variety of sensing and monitoring systems have been developed for remote monitoring in agricultural and environmental applications. As advances in electronic 


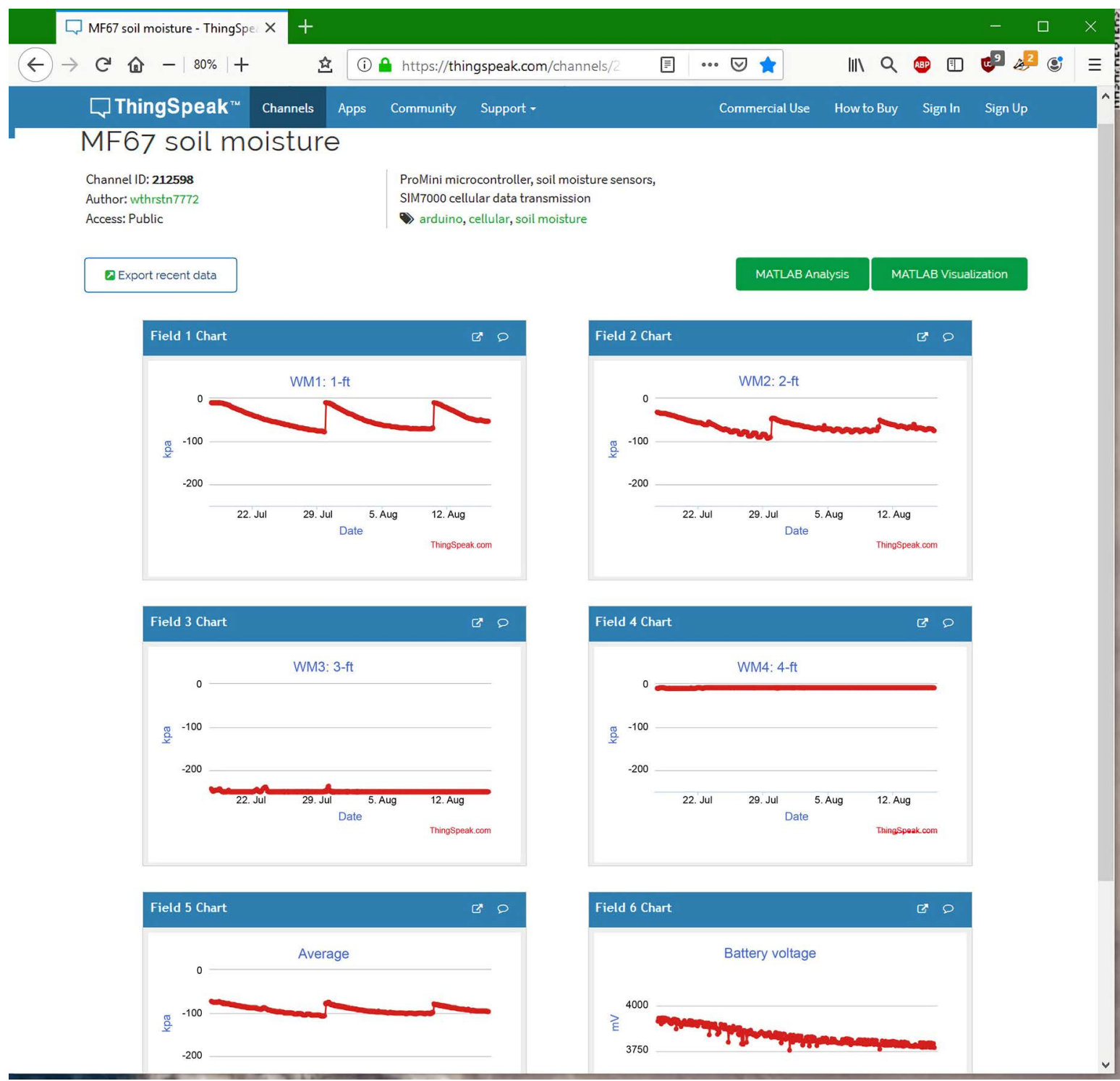

Figure 3. Monitoring system data display on a ThingSpeak webpage for a 30-day period.

hardware and cellular communications technologies occur, however, many existing monitoring systems need to be redesigned to take advantage of evolving technologies and updated cellular networks. A soil-moisture monitoring system was developed incorporating updated open-source Arduino microcontrollers and the recently introduced LTE Cat-M1 cellular network to transmit sensor measurements via the cellular network for access on an internet website. The monitoring system costs approximately US $\$ 130$ for electronic circuitry and less than US $\$ 1$ per month for cellular network access and data transmission. Data were transmitted with a $95 \%$ success rate, and the monitoring system operated continuously throughout an entire crop growing season with no battery recharge or maintenance requirements.

The soil-moisture monitoring system developed in this study can serve as a basis for developing other remote sensing or control systems. The microcontrol- 
ler and cellular shield are basic components that would serve a variety of purposes, and the software libraries and programming routines, such as those for transmitting data and low-power sleep mode, have been written and can be reused in other applications. Inexpensive, reliable, and accurate sensors are available that are designed to interface easily with programmable microcontrollers and could be of interest in the areas of agronomic and environmental monitoring, irrigation, and water use. The expanded capabilities of more-powerful microcontrollers provide more opportunities for sensor integration, increasing sophistication of data collection and analysis due to higher processing speeds and increased memory available, and longer unattended operational periods under battery-powered operation. Simple, inexpensive, and near-global access to the LTE Cat-M1 cellular network can allow users to develop and deploy unique Internet of Things applications and gain information to address specific needs.

\section{Disclaimer}

Mention of a trade name, proprietary product, or specific equipment does not constitute a guarantee or warranty by the United States Department of Agriculture and does not imply approval of the product to the exclusion of others that may be available.

\section{Conflicts of Interest}

The authors declare no conflicts of interest regarding the publication of this paper.

\section{References}

[1] Pearce, J.M. (2012) The Case for Open Source Appropriate Technology. Environment, Development and Sustainability, 14, 425-431. https://doi.org/10.1007/s10668-012-9337-9

[2] Fisher, D.K. and Gould, P.J. (2012) Open-Source Hardware is a Low-Cost Alternative for Scientific Instrumentation and Research. Modern Instrumentation, 1, 8-20. https://doi.org/10.4236/mi.2012.12002

[3] Ray, P.P. (2017) Internet of Things for Smart Agriculture: Technologies, Practices and Future Direction. Journal of Ambient Intelligence and Smart Environments, 9 , 395-420. https://doi.org/10.3233/AIS-170440

[4] Ismail, D., Rahman, M. and Saifullah, A. (2018) Low-Power Wide-Area Networks: Opportunities, Challenges and Directions. Proceedings of the Workshop Program of the 19th International Conference on Distributed Computing and Networking, Varanasi, India, 4-7 January 2018, Article No. 8. https://doi.org/10.1145/3170521.3170529

[5] Rama, Y. and Alper Özpmar, M. (2018) A Comparison of Long-Range Licensed and Unlicensed LPWAN Technologies According to Their Geolocation Services and Commercial Opportunities. 2018 18 th Mediterranean Microwave Symposium (MMS), Istanbul, Turkey, 31 October-2 November 2018, 398-403.

https://doi.org/10.1109/MMS.2018.8612009

[6] Nandhini, R., Poovizhi, S., Priyanka, J., Ranjitha, R. and Anila, S. (2017) Arduino 
Based Smart Irrigation System Using IoT. 3rd National Conference on Intelligent Information and Computing Technologies, IICT, Coimbatore.

[7] Fisher, D.K., Woodruff, L.K., Anapalli, S.S. and Pinnamaneni, S.R. (2018) OpenSource Wireless Cloud-Connected Agricultural Sensor Network. Journal of Sensor and Actuator Networks, 7, 47. https://doi.org/10.3390/jsan7040047

[8] Ramachandran, V., Ramalakshmi, R. and Srinivasan, S. (2018) An Automated Irrigation System for Smart Agriculture Using the Internet of Things. 2018 15th International Conference on Control, Automation, Robotics and Vision (ICARCV), Singapore, 18-21 November 2018, 210-215. https://doi.org/10.1109/ICARCV.2018.8581221

[9] Payero, J.O., Mirzakhani-Nafchi, A., Khalilian, A., Qiao, X. and Davis, R. (2017) Development of a Low-Cost Internet-of-Things (IoT) System for Monitoring Soil Water Potential Using Watermark 200SS Sensors. Advances in Internet of Things, 7, 71-86. https://doi.org/10.4236/ait.2017.73005

[10] Cao-Hoang, T., Tinh, T., Van, P. and Nguyen Duy, C. (2017) Design of a Cost Effective Soil Monitoring System to Support Agricultural Activities for Smallholder. Journal of Information Communication Technology and Digital Convergence, 2, 1-5.

[11] Culman, M., Portocarrero, J.M., Guerrero, C.D., Bayona, C., Torres, J.L. and de Farias, C.M. (2017) PalmNET: An Open-Source Wireless Sensor Network for Oil Palm Plantations. 2017 14th International Conference on Networking, Sensing and Control (ICNSC), Calabria, Italy, 16-18 May 2017, 783-788. https://doi.org/10.1109/ICNSC.2017.8000190

[12] Fisher, D.K., Fletcher, R.S., Anapalli, S.S. and Pringle III, H.C. (2018) Development of an Open-Source Cloud-Connected Sensor-Monitoring Platform. Advances in Internet of Things, 8, 1-11. https://doi.org/10.4236/ait.2018.81001

[13] Spinelli, G.M. and Gottesman, Z.L. (2019) A Low-Cost Arduino-Based Datalogger with Cellular Modem and FTP Communication for Irrigation Water Use Monitoring to Enable Access to CropManage. HardwareX, 6, e00066. https://doi.org/10.1016/j.ohx.2019.e00066

[14] Mangundu, E.M., Mateus, J.N., Zodi, G.A.L. and Johson, J. (2017) A Wireless Sensor Network for Rainfall Monitoring, Using Cellular Network: A Case for Namibia. 2017 Global Wireless Summit (GWS), Cape Town, 15-18 October 2017, 240-244. https://doi.org/10.1109/GWS.2017.8300469

[15] Raghava, T.K.V. and Wani, S.P. (2014) Internet Enabled Tipping Bucket Rain Gauge. International Conference on Computer Communication and Informatics, 1-5.

[16] Smith, I.G. (2012) The Internet of Things 2012: New Horizon. IERC Internet of Things European Research Cluster, Halifax, UK. 